Es ist heute fast schon selbstverständliches Wissen, $\mathrm{da} ß$ der Zellkern in Ausübung seiner genetischen Funktion "Nachrichten" an die Zelle abgibt; das soll daher hier nicht näher diskutiert werden. Auffällig scheint nur eine gewisse Uniformität solcher „Antworten" des Zellkerns, in dem offenbar die verschiedensten Anlässe wie Wachs- tum, Hormone, Viren, Ernährung, stets die prinzipiell gleiche Kausalkette - Genaktivierung $\rightarrow$ RNS-Synthese $\rightarrow$ Proteinsynthese - auszulösen scheinen. Wer hier nach molekularen Mechanismen sucht, findet sicher ein reiches Betätigungsfeld.

\title{
Literatur
}

1. Chauveau, J., Y. Moulé und C. Rouiller, Exp. Cell Res. 11, 317 (1956). - 2. Behrens, M., Hoppe-Seyler's Z. physiol. Chem. 209, 59 (1932). - 3. Siebert, G., J. G. Villalobos jr., T. S. Ro, W. J. Steele, G. Lindenmayer, H. Adams und H. Busch, J. biol. Chemistry 241, 71 (1966). - 4. Conaver, T. E. und G. Siebert, Biochim. biophysica Acta (Amsterdam) 99, 1 (1965). - 5. LANGENDorf, H., G. Siebert, I. Lorenz, R. Hannover und R. Beyer, Biochem. Z. 335, 273 (1961). - 6. SieberT, G., H. LANGENDORF, R. Hannover, D. Nitz-Litzow, B. C. Pressman und C. Moore, Hoppe-Seyler's Z. physiol. Chem., 343, 101 (1965/66). - 7. REID, E., A. A. El-Aaser, M. K. Turner und G. Siebert, HoppeSeyler's Z. physiol. Chem. 339, 135 (1964). - 8. Siebert, G., G.B. Humphrey, H. Themann und W. Kersten, Hoppe-Seyler's $Z$. physiol. Chem. 340, 51 (1965). - 9. Schuel, H. und N. G. ANDERson, J. Cell Biol. 21, 309 (1964). - 10. NeIr, M. W. und M. W. Homer, Biochem. J. 92, 217 (1964); 93, 220 (1964). - 11. Shrbko, S. und A. L. TAPper, Biochim. biophysica Acta (Amsterdam) 73, 76 (1963). - 12. Siebert, G., K. Kesselring, R. Beyer, K.-H. Bässler und B. C. Pressman, Hoppe-Seyler's Z. physiol. Chem., in Vorbereitung. - 13. Siebert, G. und G. B. Humphrey, Advances in Enzymol. 27, 239 (1965). - 14. Bakay, B., G. Siebert und S. Sorof, Cancer Res., in Vorbereitung. - 15. Robertson, J. D., Biochem. Soc. Symposia 16, 3 (1959). - 16. GrLBerT, I. G. F. und
J. M. Radley, Biochim. biophysica Acta (Amsterdam) 79, 575 (1964). - 17. Kroeger, H., Nature (London) 200, 1234 (1963). 18. Siebert, G., Biochem. Z. 334, 369 (1961). - Siebert, G., K.-H. Bässler, R. Hannover, E. Adloff und R. Beyer, Biochem. $Z$. 334, 388 (1961). - 19. KeIR, H. M., R. M. S. SMelLIE und G. Siebert, Nature (London) 196, 752 (1962). - 20. BEHKr, R. M. und W. C. Schrneider, Biochim. biophysica Acta (Amsterdam) 68, 34 (1963). - 21. Bakay, B. und S. Sorof, Cancer Res. 24, 1814 (1964). - 22. ReID, E. und G. Siebert, unveröffentlicht. - 23. KoHEN, E., G. Siebert und C. Kohen, Histochemie 3, 477 (1964). - 24. Siebert, G., I. Schade und H. ZeIdLeR, unveröffentlicht. - 25. Tsukada, K. und I. Lieberman, J. biol. Chemistry 239, 2952 (1964). - 26. Lerman, M. I., V. L. Mantieva und G. P. Georgiev, Biochimija 29, 518 (1964). - 27. Ro, T. S., M. Muramatsu und H. Busch, Biochem. biophysic. Res. Commun. 14, 149 (1964). 28. Birnstiel, M. L., E. Fleissner und E. Borek, Science (New York) 142, 1577 (1963). - 29. Сомв, D. G. und S. Katz, J. molecular Biol. 8, 790 (1964). - 30. Brandt, E. E. und F. J. Finamore, Biochim. biophysica Acta (Amsterdam) 68, 618 (1963). -31. Villalobos, jr., J. G., W. J. Steele und H. Busch, Biochem. biophysic. Res. Commun. 17, 723 (1964); Biochim. biophysica Acta (Amsterdam) 91, 233 (1964).
Professor Dr. med. G. Siebert 65 Mainz

Postfach 606

\section{Die Bestimmung der $\beta$-Glucuronidase im Serum und im Urin}

\author{
Von R. Richterich und H. Dauwalder \\ Aus dem Chemischen Zentrallaboratorium des Inselspitals (Direktor: PD Dr. R. Ricbterich) und der Kinderklinik \\ der Universität Bern (Direktor: Prof. Dr. E. Rossi)
}

(Eingegangen am 14. Juni 1965)

\begin{abstract}
Es wird eine einfache Methode zur Bestimmung $\operatorname{der} \beta$-Glucuronidase in Körperflüssigkeiten beschrieben. Als Substrat dient Phenolphthalein-Glucuronid und die Messung erfolgt durch den Nachweis des abgespaltenen Phenolphthaleins. Eine Enteiweißung ist nicht notwendig. Die Normalwerte für menschliches Serum und Urin werden in Internationalen Einheiten zusammengestellt.

A simple method for an assay of $\beta$-Glucuronidase in body fluids is described. Phenolphthalein glucuronide is used as a substrate and the liberated phenolphthalein is measured colorimetrically. The normal values for $\beta$-glucuronidase in human serum and urine are given in International Units.
\end{abstract}

Die $\quad \beta$-Glucuronidase $\quad(\beta$-D-Glucuronid-Glucuronohydrolase, EL 3.2.31 (1) katalysiert die Reaktion:

$\beta$-D-Glucuronid $+\mathrm{H}_{2} \mathrm{O} \leftrightharpoons$ Alkohol $+\mathrm{D}$-Glucuronsäure In der klinischen Chemie werden zur Bestimmung der $\beta$-Glucuronidase vor allem qwei Methoden verwendet: 1. Einsatz von Phenolphthalein-Glucuronid als Substrat und photometrische Bestimmung des freigesetzten Phenolphthaleins durch Messung der Eigenfarbe dieses Indikators im alkalischen Bereich $(2,3)$.
2. Einsatz von 6-Brom-2-Naphthyl- $\beta$-D-Glucopyroronosid als Substrat und photometrische Bestimmung der abgespaltenen aromatischen Komponente nach Uberführung in einen Azofarbstoff (4).

- Die erste Methode ist einfacher, rascher und weniger störanfällig. Wir geben ihr daher den Vorzug. Im Gegensatz zu den meisten Autoren $(3,5)$ führen wir die Bestimmung ohne vorhergehende Enteiweißung, aber nach Zentrifugation durch $(2,6)$. 


\section{Methodik}

\section{Prinzip}

1. Inkubation von Phenolphthalein-Glucuronid mit dem Untersuchungsmaterial bei $\mathrm{pH} 4,5$ und $37^{\circ}$ während $4 \mathrm{Stdn}$.

2. Unterbrechung der enzymatischen Reaktion und Alkalisierung durch Zusatz von Glykokoll-NaOH-Puffer.

3. Messung der Extinktion des freigesetzten Phenolphthaleins bei $546 \mathrm{~m} \mu$.

4. Berechnung des Resultates mit Hilfe einẹs mitgeführten Phenolphthalein-Standards.

\section{Ansatz}

Phenolphthalein-Glucuronid 3,57 mMol., pH 4,5; Citrat-Puffer $42 \mathrm{mMol} \mathrm{pH} \mathrm{4,5;} \mathrm{Analyse} 0,1 \mathrm{~m}$, Inkubationsdauer $240 \mathrm{Min}$., Inkubations-Temperatur $37^{\circ}$.

\section{Chemikalien}

Phenolphthalein-Glucuronid (freie Säure): $\mathrm{C}_{26} \mathrm{H}_{26} \mathrm{O}_{10}$; Mol.-Gew. 494,43; Phenolphthalein: $\mathrm{C}_{20} \mathrm{H}_{14} \mathrm{O}_{4}$; Mol.-Gew. 318,31.

\section{Reagenzien}

Citrat-Puffer $50 \mathrm{mMol} \mathrm{pH} \mathrm{4,5:} 28 \mathrm{~m} l \quad 0,1 m$ Citronensäure und $22 \mathrm{ml} 0,1 \mathrm{~m}$ Natrium-Citrat werden mit destilliertem Wasser auf $100 \mathrm{ml}$ gelöst.

Glykokoll-NaOH-Puffer $100 \mathrm{mMol} \mathrm{pH}$ 11,0: $51 \mathrm{ml}$ 0,1 m Glykokoll-Lösung und $0,1 \mathrm{~m}$ Natriumchlorid-Lösung werden mit $49 \mathrm{~m} l$ $0,1 \mathrm{~m}$ Natronlauge gemischt. Als Konservierungsmittel kann $1 \mathrm{ml}$ Chloroform oder Thymol-Isopropanol zugesetzt werden.

Substrat-Lösung $5 \mathrm{mMol} \mathrm{pH} \mathrm{4,5:} 247 \mathrm{mg}$ Phenolphthalein-Glucuronid mit Citrat-Puffer auf $100,0 \mathrm{ml}$ lösen. Gefroren aufbewahren.

Standard-Lösung $126 \mu \mathrm{Mol}: 4 \mathrm{mg}$ Phenolphthalein mit Äthanol auf $100,0 \mathrm{~m} /$ lösen.

Vorgeben

\begin{tabular}{lcccc}
\hline \multicolumn{1}{c}{ Ansatz in ml } & A & AL & RL & S \\
\hline Substrat-Lösung & 0,5 & - & 0,5 & - \\
Citrat-Puffer & - & 0,5 & - & 0,5 \\
Analyse & 0,1 & 0,1 & - & - \\
DM-Wasser & - & - & 0,1 & - \\
Standard-Lösung & - & - & - & 0,1
\end{tabular}

Mit Parafilm verschlossen während 240 Min. im Wasserbad bei $37^{\circ}$ inkubieren.

Glykokoll- $\mathrm{NaOH}-$

$\begin{array}{lllll}\text { Puffer } & 1,0 & 1,0 & 1,0 & 1,0\end{array}$

Gut mischen und scharf zentrifugieren und die Extinktion des Überstandes bei $546 \mathrm{~m} \mu$ gegen DM-Wasser ablesen.

\section{Berechnung}

$$
\begin{gathered}
\mathrm{IU}=\frac{\mathrm{E}(\mathrm{A})-\mathrm{A}(\mathrm{AL})-\mathrm{E}(\mathrm{RL})}{\mathrm{E}(\mathrm{S})} \cdot \mathrm{Cm}(\mathrm{S}) \cdot 10^{6} \\
\cdot \frac{1}{\mathrm{t}} \mu \mathrm{Mol} \mathrm{Min.} .^{-1} l^{-1} \\
\mathrm{IU}=\frac{\mathrm{E}(\mathrm{A})-\mathrm{A}(\mathrm{AL})-\mathrm{E}(\mathrm{RL})}{\mathrm{E}(\mathrm{S}) \cdot\left(126.10^{-6} \cdot 10^{-6}\right.} \\
\cdot \frac{1}{240} \mu \mathrm{Mol} \mathrm{Min.} .^{-1} l^{-1} \\
\mathrm{IU}=\frac{\mathrm{E}(\mathrm{A})-\mathrm{E}(\mathrm{AL})-\mathrm{E}(\mathrm{RL})}{\mathrm{E}(\mathrm{S})} \cdot 0,525 \mu \mathrm{Mol} \mathrm{Min.} .^{-1} l^{-1} \cdot
\end{gathered}
$$

\section{Spezifität}

Serum: Die Methode ist an sich spezifisch für $\beta$-Glucuronidase. Es ist jedoch noch nicht geklärt, ob in den Körperflüssigkeiten nur ein einziges Enzym dieses Typs vorkommt oder ob mehrere Enzym-Species mit ähnlicher katalytischer Wirkung vorkommen (Heterogenität).

Urin: $\mathrm{Ob}$ die im Urin vorkommende $\beta$-Glucuronidase ein einheitliches Enzym darstellt oder ob verschiedene molekulare Species vorkommen, ist bisher nicht geklärt.

\section{Stabilität}

Serum: Die Serum- $\beta$-Glucuronidase gehört $\mathrm{zu}$ den stabilsten Serum-Enzymen. In gefrorenem Zustand bleibt ihre Aktivität unbeschränkt haltbar, bei Zimmertemperatur ist nach 48 Stdn. mit einem Aktivitätsverlust von etwa $10 \%$ zu rechnen.

Urin: Boyland und Mitarbeiter (11) fanden keine Aktivitäts-Einbuße der Urin- $\beta$-Glucuronidase beim Aufbewahren bei Zimmertemperatur oder $5^{\circ}$ während 6 Tagen. Dennoch empfiehlt sich ein Zusatz von Thymol-Benzol (10) oder Thymol-Isopropanol (15) als Ưrin-Konservierungsmittel.

\section{Ergebnisse}

\section{Normalwerte}

Serum

\begin{tabular}{lrlr}
\hline Probanden & N & IU $\left(\mu \mathrm{Mol}\right.$ Min..$\left.^{-1} l^{-1}\right)$ & $\begin{array}{r}\text { Litera- } \\
\text { turzitat }\end{array}$ \\
\hline Männer & 16 & $\left.0-0,095^{1}\right)$ & 3 \\
& 10 & $\left.0,118 \pm 0,12^{2}\right)$ & 8 \\
& 6 & $\left.0,164(0,131-0,288)^{3}\right)$ & 9 \\
& 18 & $\left.0,120(0,030-0,270)^{4}\right)$ & 13 \\
Frąuen & 16 & $\left.0-0,120^{1}\right)$ & $3 \cdot$ \\
& 10 & $0,168 \pm 0,132)$ & 8 \\
& 14 & $\left.0,164(0,052-0,248)^{3}\right)$ & 9 \\
& 18 & $\left.0,120(0,030-0,270)^{4}\right)$ & 13 \\
Gravide, 1. Trimester & 2 & $\left.0,186(0,136-0,236)^{3}\right)$ & 9 \\
Gravide, 2. Trimester & 10 & $\left.0,335(0,157-0,524)^{3}\right)$ & 9 \\
Gravide, 3. Trimester & 21 & $\left.0,471(0,157-0,812)^{3}\right)$ & 9 \\
Gravide, Wehenbeginn & 27 & $\left.0,453(0,262-0,706)^{3}\right)$ & 9 \\
Gravide, Geburt & 24 & $\left.0,488(0,262-1,020)^{3}\right)$ & 9 \\
Wöchnerinnen, & & & \\
$\quad$ 7 Tage post partum & 20 & $\left.0,208(0,162-0,288)^{3}\right)$ & 9 \\
Menopause & 20 & $0,275($ & \\
Nabelschnurblut & 46 & $\left.0,181(0,116-0,294)^{3}\right)$ & 9 \\
\hline
\end{tabular}

1) Statistisch nicht ausgewerteter Streubereich.

$\stackrel{2}{2} \overline{\mathrm{x}} \pm 2 \mathrm{~s}$.

3) $\overline{\mathrm{X}}$ (statistisch nicht ausgewerteter Streubereich).

4) $\overline{\mathbf{x}}$ (graphisch ermittelter $2-98 \%$ Bereich).

\section{Urin}

Alle folgenden Angaben wurden auf Grund von Bestimmungen mit Phenolphthalein-Glucuronid als Substrat erhalten und in IU, d. h. $\mu \mathrm{Mol} \mathrm{Min.} .^{-1}\left(37^{\circ}\right)$ umgerechnet. 


\section{Probanden}

N IU $\left(\mu \mathrm{Mol} \mathrm{Min.}^{-1} l^{-1}\right)$ Literaturzitat

Kontrollen

Kontrollen (Urin steril) 25

Kontrollen (Urin infiziert) 16

Kontrollen

Kontrollen

\begin{tabular}{llll}
\hline & & IU $\left(\mu\right.$ Mol Min. ${ }^{-1}$ pro 24 Stdn. $)$ \\
\hline Kontrollen & 10 & $-0,147$ & 10 \\
Kontrollen & 24 & $\left.0,105(-0,157)^{1}\right)$ & 12 \\
\hline
\end{tabular}

1) $\bar{x}$ (Streubereich).

2) $\overline{\bar{x}}$ (graphisch ermittelter 2-98\% Bereich).

\section{Diskussion}

Die optimalen Bedingungen dieses Enzymes in den Körpersäften sind noch wenig untersucht. Als Optimum für das Serum-Enzym wird pH 4,1-5,2 angegeben (6). Unter den angegebenen Bedingungen gilt die EnzymUmsatz- und Zeit-Umsatz-Regel für den normalen und pathologischen Bereich.

Bleibt die Analyse nach der Zentrifugation noch trübe, so ist die Bestimmung zu wiederholen. Nach der Inkubation werden alle Ansätze mit 1,0 ml 0,33 $n$ Perchlorsäure enteiweißt, der Überstand neutralisiert und dann $1 \mathrm{~m} l$ des Überstandes mit Glykokoll-NaOH-Puffer versetzt. Bei der Enteiweißung ist mit Verlusten an Phenolphthalein, d. h. mit einer reduzierten Enzym-Aktivität zu rechnen (6).

Erythrocyten enthalten keine $\beta$-Glucuronidase, d. h. geringe Hämolyse stört bei der Bestimmung nicht (3).

Die meisten Aktivitäts-Angaben in der Literatur sind in Fishman-Einheiten (3), FU, ausgedrückt. 1 FU entspricht derjenigen Enzym-Menge, die in 1 Std. $1 \mu \mathrm{g}$ Phenolphthalein aus Phenolphthalein-Glucuronid freisetzt. Als Bezugsgröße wird in der Regel $1 \mathrm{ml}$ (Urin) oder $100 \mathrm{ml}$ (Serum) verwendet. Der Umrechnungs-Faktor von FU in IU lautet:

$1 \mathrm{FU}=\frac{1}{\mathrm{MG}} \cdot \frac{1}{\mathrm{t}} \cdot 1000 \mu \mathrm{Mol} \mathrm{Min}^{-1} l^{-1}$ (Urin), d. h.
$1 \mathrm{FU}=\frac{1}{318,31} \cdot \frac{1}{60} \cdot 1000 \mu \mathrm{Mol} \mathrm{Min.} .^{-1} l^{-1}$ (Urin) oder

$1 \mathrm{FU}=0,0523 \mu \mathrm{Mol} \mathrm{Min.} .^{-1} l^{-1}$ (Urin).

Für Serum (100 ml Bezugsgröße) heißt die Formel $1 \mathrm{FU}=0,000523 \mu \mathrm{Mol} \mathrm{Min.} .^{-1} l^{-1}$.

Im Prinzip kann die Bestimmung auch nach dem Absolut-Meßprinzip durchgeführt werden, d. h. bei der Verwendung geeichter Pipetten, von Küvetten mit $1 \mathrm{~cm}$ Schichtdicke und monochromatischem Licht von $546 \mathrm{~m} \mu$ kann auf das Mitführen eines Standards verzichtet werden. Von einem solchen Vorgehen ist im vorliegenden Falle jedoch abzuraten, da geringe $\mathrm{pH}-\mathrm{Ver}-$ schiebungen zu großen Fehlern führen können. Als molarer. Extinktions-Koeffizient für Phenolphthalein in alkalischer alkoholischer Lösung wird 33550 angegeben (7).

Menschliches Serum hat eine gewisse Hemmwirkung auf die Aktivität der $\beta$-Glucuronidase (8). Unter den vorliegenden Meßbedingungen fühtt dieses jedoch nicht zu einer Störung der Bestimmung.

Die Mehrzahl der Autoren konnten im Urin keinen Inhibitor der $\beta$-Glucuronidase nachweisen $(11,13)$. Der besonders von italienischen Autoren beobachtete Verdünnungs-Effekt (14) ist wahrscheinlich durch die Versuchs-Anordnung bedingt.

Die Enzym-Ausscheidung im Urin ist starken Tagesschwankungen unterworfen (11). Eine Beziehung zur Zellzahl ist nicht nachzuweisen, obschon das Urin-Sediment mehr $\beta$-Glucuronidase als der abzentrifugierte Überstand enthält (11).

Der Ursprung der Urin- $\beta$-Glucuronidase ist noch ungenügend geklärt. Ein Teil dürfte aus dem Plasma stammen, ein weiterer Teil aus den ableitenden Harnwegen. Dafür sprechen einerseits die schlechte Korrelation mit der Kreatinin-Ausscheidung $(12,13)$, andererseits die Beobachtung, daß Blasenspülwasser ansehnliche Enzymmengen enthält (12).

Die vorliegenden Untersuchungen wurden mit Unterstützung des „Schweizerischen Nationalfond zur Förderung der wissenschaftlichen Forschung" durchgeführt.

\section{Literatur}

1. Report of the Commission on Enzymes of the International Union for Biochemistry. Revised edition. Elsevier Publ. Comp., Amsterdam-London-New York (1964). - 2. TalalAy, P., W. H. FrshMAN und C. Huggins, J. biol. Chemistry 166, 757 (1956). - 3. Fishman, W. H., B. SpRINGER und R. Brunettr, J. biol. Chemistry 173, 449 (1948). - 4. Goldbarg, J. A., E. P. Pineda, B. M. Banks und A. M. Rutenburg, Gastroenterology, Baltimore 36, 193 (1959). - 5. Fishiman, W. H. in: Methoden der enzymatischen Analyse, herausgeg. von H. U. Bergmeyer. S. 869. Verlag Chemie GmbH, Weinheim, Bergstr. (1962). - 6. Goldstern, G.; Clin. Chem. (New York) 7, 136 (1961). - 7. Allen, J., B. Gartside und C. A. Johnson, J. Pharmacy Pharmacol. 14, 73 (1962). -
8. Dohrmann, R. E. und H. J. Uhles, Klin. Wschr. 41, 527 (1963). - 9. Kasdon, S. C., P. Romano und A. Hatzimichaed, Obstetrics and Gynecology (New York) 15, 367 (1960). - 10. MeLrcow, M. M., A. C. Uson und R. Lipron, J. Urol., Baltimore 86, 89 (1961). - 11. Boyland, E., D. M. Wallace und D. C. Williams, Brit. J. Cancer 9, 62 (1955). - 12. KERR, W. K., M. BARKIN, J. D'Aloisio und Z. Menczyk, Cancer 16, 633 (1963). - 13. Appert, O. und R. Richterich, in Vorbereitung. - 14. Mattea, E., E. Pietra und M. R. Viola, Tumori 45, 402, 229, 239 (1959). - 15. Richterich, R., Klinische Chemie, Theorie und Praxis. S. Karger AG., BaselNew York (1964).
Priv.-Doz. Dr. R. Richterich Med.-Chem. Institut der Universität

Bern (Schweiz), Bühlstr. 28 